\title{
Extremely low-frequency spectroscopy in low-field nuclear
}

magnetic resonance

G. Pileio, M. Carravetta and M.H. Levitt

School of Chemistry, University of Southampton, Southampton UK SO17 1BJ.

E-mail:marina@soton.ac.uk

Please cite this paper as:

Phys. Rev. Lett. 103, 2009,(083002)

The publisher's version of this paper is available here:

http://dx.doi.org/10.1103/PhysRevLett.103.083002

\section{Related article by Dr Marina Carravetta can be found below:}

G. Pileio, M. Carravetta and M.H. Levitt, (2010) Storage of nuclear magnetization as longlived singlet order in low magnetic field. Proc. Nat. Acad. USA 107, 17135-17139.

(doi:10.1073/pnas.1010570107). 


\title{
Extremely Low-Frequency Spectroscopy in Low-Field Nuclear Magnetic Resonance
}

\author{
Giuseppe Pileio, Marina Carravetta, and Malcolm H. Levitt* \\ School of Chemistry, Southampton University, SO17 1BJ, UK
}

(Dated: July 14, 2009)

\begin{abstract}
We demonstrate a new phenonemon in nuclear magnetic resonance spectroscopy, in which nuclear spin transitions are induced by radiofrequency irradiation at extremely low frequencies (of the order of a few Hz). Slow Rabi oscillations are observed between spin states of different exchange symmetry. These "forbidden" transitions are rendered weakly-allowed by differential electronic shielding effects on the radiofrequency field. We generate coherence between the singlet and triplet states of ${ }^{15} \mathrm{~N}$ labelled nitrous oxide in solution, and estimate the scalar coupling between the two ${ }^{15} \mathrm{~N}$ nuclei with a precision of a few $\mathrm{mHz}$.
\end{abstract}

In nuclear magnetic resonance, radiofrequency irradiation resonant with the nuclear Zeeman splitting is used to induce nuclear spin transitions. In conventional highfield NMR, the resonant frequency is typically hundreds of $\mathrm{MHz}$, while in NMR at very low field, such as NMR in the earth's field $[1,2]$, the resonant frequency may be a few $\mathrm{kHz}$. Here we demonstrate resonant nuclear spin effects at still lower frequencies. We show that electromagnetic irradiation in the extremely low frequency (ELF) region of the radiofrequency spectrum, of the order of a few $\mathrm{Hz}$, can induce nuclear spin transitions between states of different spin exchange symmetry. The transitions are weakly-allowed because of differential chemical shielding of the ELF field.

Our demonstration system is doubly ${ }^{15} \mathrm{~N}$-labelled nitrous oxide $\left({ }^{15} \mathrm{~N}_{2} \mathrm{O}\right.$, dinitrogen monoxide), which supports a low-field nuclear singlet state with a lifetime of tens of minutes in solution [3]. Nitrous oxide is non-toxic, dissolves in many important solvents such as water and oil, and is widely used in medicine [4] and food processing. The extraordinarily long singlet lifetime of ${ }^{15} \mathrm{~N}_{2} \mathrm{O}$ suggests applications to the characterization of slow diffusion and flow in medical and industrial materials [3]. The ELF spectroscopy of ${ }^{15} \mathrm{~N}_{2} \mathrm{O}$ creates further possibilities for the scientific applications of this substance.

The two ${ }^{15} \mathrm{~N}$ nuclei of ${ }^{15} \mathrm{~N}_{2} \mathrm{O}$ are in chemically inequivalent sites, with a chemical shift difference of $\Delta \delta=82.3$ ppm and a scalar spin-spin coupling $J_{N N} \simeq 8 \mathrm{~Hz}[3]$. In zero magnetic field, the pair of ${ }^{15} \mathrm{~N}$ nuclei become magnetically equivalent, with nuclear eigenstates given by the singlet state and the three triplet states, defined as follows:

$$
\begin{array}{ll}
\left|S_{0}\right\rangle=\frac{1}{\sqrt{2}}(|\alpha \beta\rangle-|\beta \alpha\rangle) & \left|T_{1}\right\rangle=|\alpha \alpha\rangle \\
\left|T_{0}\right\rangle=\frac{1}{\sqrt{2}}(|\alpha \beta\rangle+|\beta \alpha\rangle) & \left|T_{-1}\right\rangle=|\beta \beta\rangle
\end{array}
$$

Spin- $1 / 2$ states with angular momentum $\pm(1 / 2) \hbar$ along the $\mathrm{z}$-axis of the (arbitrarily defined) laboratory reference frame are denoted $\alpha$ and $\beta$ respectively. In zero magnetic field, the three degenerate triplet states are higher in energy than the singlet state by the singlet-triplet splitting $2 \pi \hbar J_{N N}$. Perturbation of the singlet-triplet splitting is very small if the low-field chemical shift difference (in $\mathrm{Hz}$ ) is less than the scalar spin-spin coupling, as is the case for the experiments described below.

The three triplet populations mutually interconvert with the conventional spin-lattice relaxation time constant $T_{1}$. The singlet-triplet interconversion, on the other hand, is an order of magnitude slower, since many common relaxation mechanisms are ineffective [3, 5-16]. In the case of ${ }^{15} \mathrm{~N}_{2} \mathrm{O}$, the time constant $T_{S}$ for singlet-triplet inter-conversion may be as long as 26 minutes [3].

The singlet state is antisymmetric, and the triplet states are symmetric, with respect to exchange of the two nuclei. Singlet-triplet transitions are therefore symmetry-forbidden under any exchange-symmetric external perturbation. Conventional spin dynamical theory assumes that the interaction of a spin system with an applied radiofrequency field is exchange-symmetric, i.e. exactly the same for all resonant spins [17, 18]. This assumption is plausible since each molecule is much smaller than the length scale of radiofrequency field variations. However, this assumption neglects the shielding effects of the local molecular electrons on the applied radiofrequency field. Chemical shift effects are very well known for the strong static magnetic fields used in conventional high-field NMR. However, the influence of electronic shielding on the applied radiofrequency field has been consistently neglected. This is reasonable, since such effects are extremely small, as discussed below. Nevertheless, in the context of low-field NMR, the minute radiofrequency chemical shift effects render singlet-triplet transitions weakly allowed.

Consider, for example, a weak magnetic field $B_{z}$ along the z-axis of the laboratory reference frame. The nuclear spin Hamiltonian describing the interaction of the 2-spin system with the magnetic field is given by

$$
H_{z}=-\gamma B_{z}\left\{\left(1+\delta_{1}\right) I_{1 z}+\left(1+\delta_{2}\right) I_{2 z}\right\}
$$

where the spin angular momentum operators are denoted $I_{1}$ and $I_{2}$, the chemical shifts are $\delta_{1}$ and $\delta_{2}$, and $\gamma$ is the nuclear magnetogyric ratio. The off-diagonal matrix element connecting the singlet state and the central triplet 


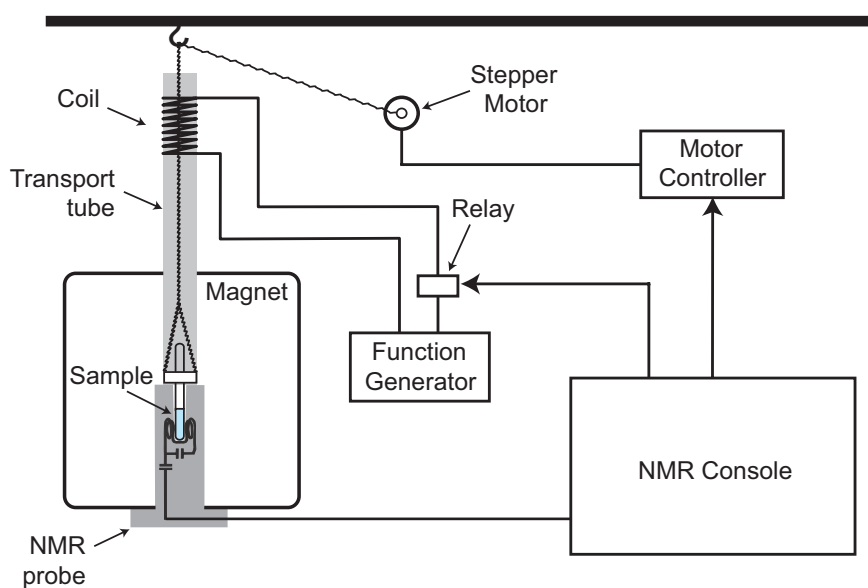

FIG. 1: Equipment used to perform ELF NMR experiments.

state is given by

$$
\left\langle S_{0}\left|H_{z}\right| T_{0}\right\rangle=-\frac{1}{2} \gamma \Delta \delta B_{z}
$$

where $\Delta \delta=\delta_{1}-\delta_{2}$. If the field $B_{z}$ is periodically modulated, so that one of its Fourier components is resonant with the singlet-triplet splitting, Rabi oscillations between the states $\left|S_{0}\right\rangle$ and $\left|T_{0}\right\rangle$ are induced. If the modulated magnetic field has the form $B_{z}(t)=$ $B_{\mathrm{ELF}} \cos \left(\omega_{\mathrm{ELF}} t\right)$ where $B_{\mathrm{ELF}}$ is the peak ELF field amplitude, and the modulation frequency $\omega_{\mathrm{ELF}}$ exactly matches the singlet-triplet splitting, the Rabi frequency for singlet-triplet nutation is given by

$$
\omega_{\mathrm{Rabi}}^{\mathrm{ST}}=\left|\frac{1}{2} \gamma B_{\mathrm{ELF}} \Delta \delta\right|
$$

This corresponds to the Larmor frequency in the ELF field, scaled by the minuscule chemical shift difference $\Delta \delta=82.3 \mathrm{ppm}$. For practical ELF fields, the singlettriplet Rabi frequency is only a fraction of $1 \mathrm{~Hz}$.

The ELF resonance condition is extremely narrow. Nevertheless, the resonance is readily observed since ordinary laboratory oscillators provide $\mathrm{mHz}$ frequency stability, and the energy difference between the singlet state $\left|S_{0}\right\rangle$ and the central triplet state $\left|T_{0}\right\rangle$ is highly insensitive to magnetic field variations.

The apparatus used to detect ELF singlet-triplet transitions is shown in Fig. 1. This is based on a conventional high-field NMR magnet $\left(B^{0}=7.05 \mathrm{~T}\right)$ equipped with conventional radiofrequency electronics, but with the facility of transporting the sample in and out of the magnet using a stepper motor attached to a piece of string, passing over a hook in the laboratory ceiling. At the top end of its trajectory, the sample enters a 300-turn copper solenoid, located about $1 \mathrm{~m}$ above the top of the NMR magnet, and with its axis parallel to the fringe field of the magnet. The peak field generated by the ELF coil was $B_{\mathrm{ELF}} \simeq 0.6 \mathrm{mT}$. The ELF pulses were generated by gating the sinusoidal output of a commercial signal generator

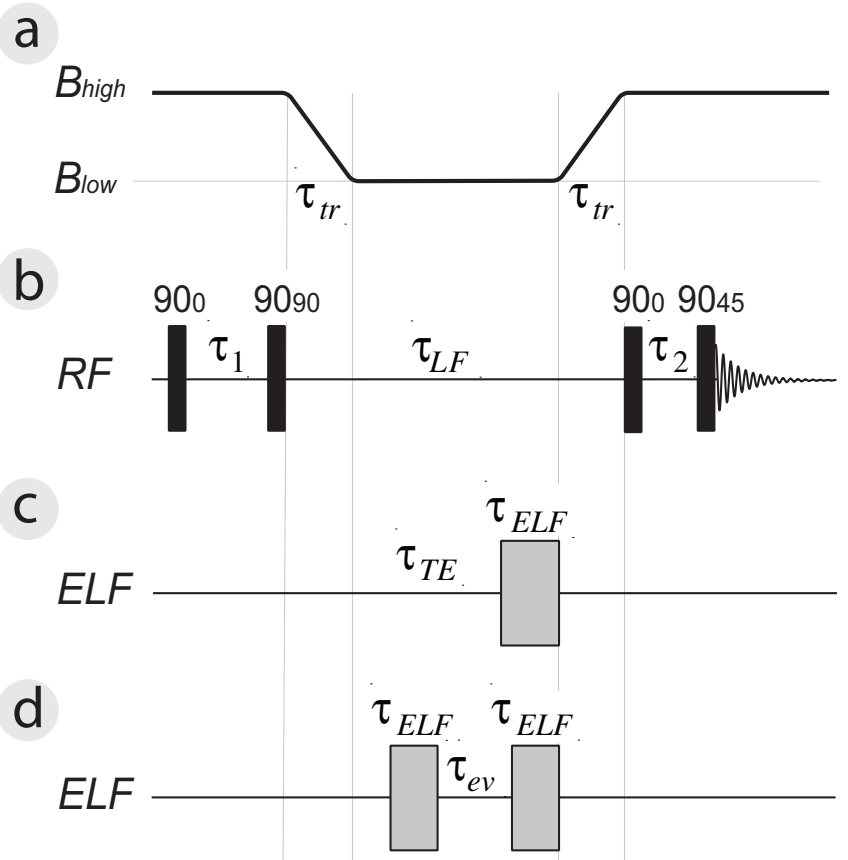

FIG. 2: Experimental timing sequences. (a) Magnetic fields, involving transport of the sample from the high magnetic field $B_{\text {high }}$ to the low magnetic field $B_{\text {low }}$, and back again. The transport time is $\tau_{\mathrm{tr}}$. (b) Radiofrequency fields, resonant with the ${ }^{15} \mathrm{~N}$ nuclei in $B_{\text {high }}$. The pulse flip angles and $\mathrm{rf}$ phases are given in degrees. The time spent in $B_{\text {low }}$ is $\tau_{\mathrm{LF}}$. (c) ELF pulse sequence for observing singlet-triplet nutation. After a time $\tau_{\mathrm{TE}}$ to allow triplet equilibration, a resonant ELF pulse is applied of duration $\tau_{\mathrm{ELF}}$. (d) ELF pulse sequence for observing the precession of singlet-triplet coherence. Two ELF pulses, with the same phase, are separated by a variable evolution interval $\tau_{\text {ev }}$. The ELF sequences are executed in parallel with sequences (a) and (b). The timing sequences are not to scale: the low-field pulses are about 4 orders of magnitude longer than the high-field ones.

using a mechanical relay, controlled by the spectrometer software.

The sample used for all experiments consisted of ${ }^{15} \mathrm{~N}_{2} \mathrm{O}$ dissolved in locally-produced Calabrian olive oil at a pressure of $\sim 3$ bar.

The timing sequences used for ELF experiments are shown in Fig. 2. Frames (a) and (b) indicate the timing sequences for a measurement of singlet relaxation time $T_{S}$, as described in ref. [3]. The sample is allowed to equilibrate in high magnetic field for 200 seconds before a sequence of two $90^{\circ}$ radiofrequency pulses, resonant with the ${ }^{15} \mathrm{~N}$ Larmor frequency, is applied. The pulse sequence timings and relative phases are chosen so as to invert the magnetization of ${ }^{15} \mathrm{~N}$ nuclei at only one of the two chemically-distinct sites. The sample is then winched out of the NMR magnet. As described in ref. [3], this causes a singlet-triplet population difference to be generated by adiabatic transport of the sample out of the magnetic field. The sample is left in low magnetic field 
a

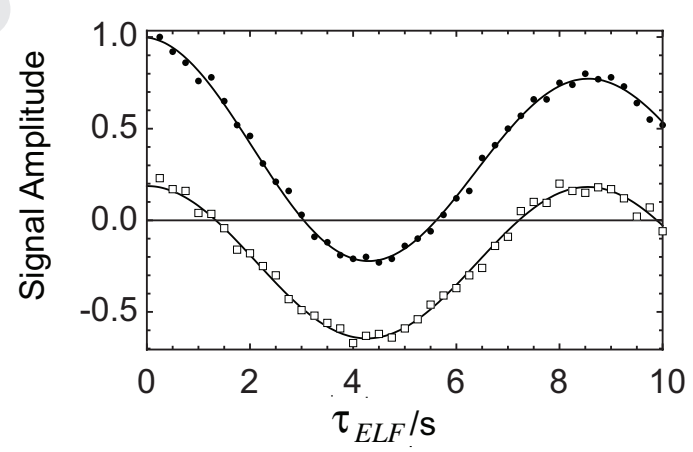

b

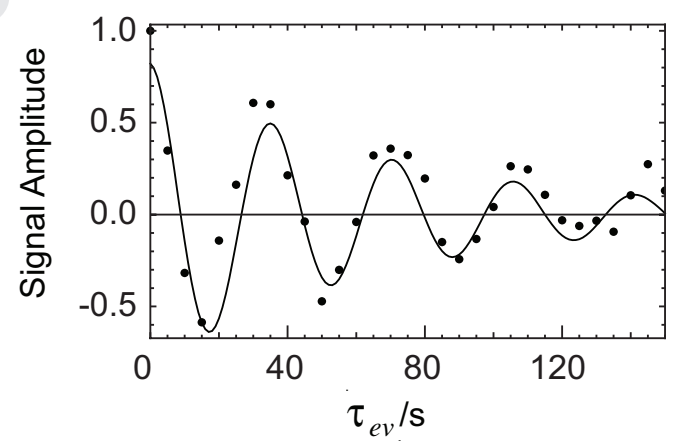

FIG. 3: Experimental peak amplitudes plotted against the timing intervals of ELF pulse experiments. (a) Singlet-triplet nutation experiment, varying the duration $\tau_{\mathrm{ELF}}$ of the ELF pulse in Fig. 2c. (b) Singlet-triplet coherence evolution experiment, varying the duration $\tau_{\mathrm{ev}}$ of the evolution interval in Fig. 2d, with the two ELF pulse durations fixed to $\tau_{\mathrm{ELF}}=2.15 \mathrm{~s}$. Black circles and white boxes refer to the amplitudes of the two components of the high-field doublet in the ${ }^{15} \mathrm{~N}_{2} \mathrm{O}$ spectrum. The solid lines are best fits to exponentiallydecaying cosine functions with an added offset from zero. Experiments were performed on a sample of ${ }^{15} \mathrm{~N}_{2} \mathrm{O}$ dissolved in locally-produced Calabrian olive oil using the following timings: $\tau_{1}=198 \mu \mathrm{s}, \tau_{2}=99 \mu \mathrm{s}, \tau_{\mathrm{tr}}=10 \mathrm{~s}$ and $\tau_{\mathrm{TE}}=60 \mathrm{~s}$. The ELF irradiation frequency was $\omega_{\mathrm{ELF}} / 2 \pi=8.693 \mathrm{~Hz}$ in all cases.

for an interval $\tau_{\mathrm{LF}}$ before the sample is let down into the magnet, a further two-pulse RF sequence is applied, and the NMR signal is detected. In the current case, measurement of the NMR signals for a set of different low-field intervals $\tau_{\text {LF }}$ provided the following estimation of the singlet relaxation time: $T_{S}=1585 \pm 57 \mathrm{~s}$, consistent with earlier experiments [3].

The timing sequence in Fig. 2c demonstrates the phenomenon of ELF-induced singlet-triplet nutation. The sample is left in the low-field region for a "triplet equilibration interval" $\tau_{\mathrm{TE}}=60$ seconds. This allows the populations to redistribute within the triplet manifold, while maintaining the singlet-triplet population difference [3]. This protocol generates a more reproducible starting point for the singlet-triplet nutation procedure, as will be described in detail elsewhere.

Singlet-triplet nutation is observed by applying an ELF pulse of duration $\tau_{\text {ELF }}$, with a frequency matching the singlet-triplet splitting. The sample is transported back into the high-field region, and the NMR signal generated by a further sequence of two $90^{\circ}$ pulses. Fourier transformation of the signal generates the NMR spectrum. The experiment is repeated for a set of different pulse durations $\tau_{\text {ELF }}$. The amplitudes of two high-field NMR peaks are plotted as a function of the ELF pulse duration $\tau_{\mathrm{ELF}}$ in Fig. 3a.

This plot shows clear Rabi oscillations indicating the coherent driving of the singlet-triplet transitions by the extremely low-frequency field. The Rabi oscillations induced by the ELF pulse are extraordinarily slow: The singlet-triplet Rabi frequency is of the order of $\omega_{\mathrm{Rabi}}^{\mathrm{ST}} / 2 \pi=116 \mathrm{mHz}$, corresponding to a $90^{\circ}$ singlettriplet pulse of duration 2.15 seconds. This is consistent with the known chemical shift difference $\Delta \delta=82.3 \mathrm{ppm}$ and a peak ELF field of $0.65 \mathrm{mT}$. The asymmetric pattern of peak amplitudes is consistent with spin-dynamical theory, as will be described elsewhere.

In principle, singlet-triplet nutation could also be induced by an unmodulated magnetic field pulse, or a sudden magnetic field step [19]. However, the field changes required in those methods are much stronger and require specialized hardware.

A resonant ELF pulse induces a rotation in the singlettriplet subspace through the singlet-triplet flip angle defined $\beta_{\mathrm{ST}}=\omega_{\mathrm{Rabi}}^{\mathrm{ST}} \tau_{\mathrm{ELF}}$. For example, a $180^{\circ} \mathrm{ELF}$ pulse, for which $\beta_{\mathrm{ST}}=\pi$, interchanges the populations of $\left|S_{0}\right\rangle$ and $\left|T_{0}\right\rangle$. A $90^{\circ}$ ELF pulse, for which $\beta_{\mathrm{ST}}=\pi / 2$, transforms the singlet state $\left|S_{0}\right\rangle$ into a superposition of $\left|S_{0}\right\rangle$ and $\left|T_{0}\right\rangle$. If a $90^{\circ}$ ELF pulse is applied to a spin ensemble displaying a singlet-triplet population difference, a coherent superposition of singlet and triplet states is induced. This is called singlet-triplet coherence.

Fig. $2 \mathrm{~d}$ shows the procedure for studying the free evolution of singlet-triplet coherence. Two $90^{\circ}$ ELF pulses are separated by a variable evolution interval $\tau_{\text {ev }}$. Incrementation of the evolution interval $\tau_{\text {ev }}$ generates prominent oscillations in the NMR signal, as shown in Fig. 3b. These oscillations may be fitted to a damped sinusoidal oscillation with frequency $50.8 \pm 0.3 \mathrm{mHz}$.

Since the ELF pulses are phase-coherent at the ELF carrier frequency, the observed oscillation frequency is given by $\left|\omega_{\mathrm{ST}}-\omega_{\mathrm{ELF}}\right|$, where $\omega_{\mathrm{ST}}$ is the singlet-triplet splitting in angular frequency units, and $\omega_{\mathrm{ELF}}$ is the angular frequency of the ELF oscillator. A second experiment, with a slightly different ELF frequency, was performed in order to determine whether the singlet-triplet frequency was larger or smaller than the ELF carrier frequency. In this way, it was possible to determine the singlet-triplet splitting to be $\omega_{\mathrm{ST}} / 2 \pi=8.695 \pm 0.001 \mathrm{~Hz}$ in angular frequency units. The decay time constant for the singlet-triplet coherence was determined to be $T_{2}^{\mathrm{ST}}=77$ $\pm 10 \mathrm{~s}$.

In zero magnetic field, the singlet-triplet splitting, ex- 
pressed in frequency units, corresponds exactly to the spin-spin coupling, i.e. $\omega_{S T}=2 \pi J_{N N}$. However, in our experiments, the fringe field of the NMR magnet at the centre of the ELF coil was estimated to be $B_{\text {low }} \simeq$ $2.0 \pm 0.1 \mathrm{mT}$ by using a Hall effect sensor. This finite ambient field leads to small second-order chemical shift effects. The singlet-triplet splitting in this regime is given by

$$
\omega_{S T} \simeq 2 \pi J_{N N}+\frac{\left(\gamma B_{\mathrm{low}} \Delta \delta\right)^{2}}{4 \pi J_{N N}}
$$

When this small shift is taken into account, the Jcoupling between the two ${ }^{15} \mathrm{~N}$ nuclei was determined to be $J_{N N}=8.665 \pm 0.004 \mathrm{~Hz}$, where the error margin takes into account the uncertainty in the chemical shift values, the magnetic field inside the ELF coil, and the measured singlet-triplet splitting.

The determination of a nuclear spin-spin coupling with such accuracy is possible because of the following factors: 1. Magnetic field inhomogeneity, which plays a strong role in conventional high-field NMR, has almost no effect on the singlet-triplet splitting; 2 . The decay of the singlet-triplet coherence is very slow, in part because of the long singlet lifetime; 3 . Even the diffusion of the molecules into sample regions that experience different magnetic fields has a negligible effect.

We have used singlet-triplet coherence to investigate whether $J_{N N}$ depends on the solvent in which ${ }^{15} \mathrm{~N}_{2} \mathrm{O}$ is dissolved. Important components of olive oil include the triglyceride esters of oleic acid (9Z-octadecenoic acid) and linoleic acid (9Z, 12Z-octadecadienoic acid). We dissolved ${ }^{15} \mathrm{~N}_{2} \mathrm{O}$ in separate samples of oleic and linoleic acid and measured the singlet-triplet oscillation frequency in both cases. The $J_{N N}$ couplings between the ${ }^{15} \mathrm{~N}$ nuclei were found to be $8.727 \pm 0.004 \mathrm{~Hz}$ and $8.698 \pm 0.004$ $\mathrm{Hz}$ for oleic acid and linoleic acid solutions respectively. These small but signicant differences suggest that the accurate measurement of J-couplings in low magnetic field can convey information on the chemical environment of the nitrous oxide.

The extremely long-lived singlet of ${ }^{15} \mathrm{~N}_{2} \mathrm{O}$ should allow hyperpolarized nuclear spin order, such as that generated by dynamic nuclear polarization (DNP) [20], to be transported through the sample with relatively little loss of order. The experiments described here shows that this use of the singlet may be combined with singlet-triplet interconversion by applying resonant ELF fields of relatively low amplitude. Since the triplet states are magnetic, they respond to applied magnetic field gradients, and may therefore be used for spatial characterization.

In summary, we have demonstrated a new form of nuclear magnetic resonance spectroscopy, involving irradiation in the extremely low frequency (ELF) region of the electromagnetic spectrum. The ELF pulses induce extremely slow Rabi oscillations between states of different spin exchange symmetry. In the case of ${ }^{15} \mathrm{~N}$-labelled ni- trous oxide, the ELF pulses induce singlet-triplet population exchange, and excite singlet-triplet spin coherence, which oscillates at a characteristic and extremely well defined frequency. Furthermore, the ELF pulses may be used to exchange spin order at will between magnetic triplet states with conventional relaxation properties, and non-magnetic singlet states with exceptionally long lifetimes. The phenomenon is not restricted to ${ }^{15} \mathrm{~N}$ labelled nitrous oxide and should be observable for many coupled nuclear spin species in low magnetic field.

We thank O. G. Johannessen, A. Glass, R. Dalley, J. James, L. Mulholland and P. Trye for instrumental help, and $\operatorname{EPSRC}(\mathrm{UK})$ for funding. MC would like to thank the Royal Society for a University Research Fellowship.

* mhl@soton.ac.uk

[1] S. Appelt, F. W. Häsing, H. Kühn, U. Sieling, and B. Blümich, Chem. Phys. Lett. 440, 308 (2007).

[2] S. Appelt, H. Kühn, F. W. Häsing, and B. Blümich, Nature Phys. 2, 105 (2006).

[3] G. Pileio, M. Carravetta, E. Hughes, and M. H. Levitt, J. Am. Chem. Soc. 130, 12582 (2008).

[4] R. J. Traystman, J. Appl. Physiol 97, 1601 (2004).

[5] M. Carravetta, O. G. Johannessen, and M. H. Levitt, Phys. Rev. Lett. 92, 153003 (2004).

[6] M. Carravetta and M. H. Levitt, J. Am. Chem. Soc. 126, 6228 (2004).

[7] S. Cavadini, J. Dittmer, S. Antonijevic, and G. Bodenhausen, J. Am. Chem. Soc. 127, 15744 (2005).

[8] M. Carravetta and M. H. Levitt, J. Chem. Phys. 122, 214505 (2005)

[9] K. Gopalakrishnan and G. Bodenhausen, J. Magn. Reson. 182, 254 (2006).

[10] G. Pileio, M. Concistré, M. Carravetta, and M. H. Levitt, J. Magn. Reson. 182, 353 (2006).

[11] P. Sarkar, P. Ahuja, D. Moskau, P. R. Vasos, and G. Bodenhausen, ChemPhysChem 8, 2652 (2007).

[12] G. Pileio and M. H. Levitt, J. Magn. Reson. 187, 141 (2007).

[13] E. Vinogradov and A. K. Grant, J. Magn. Reson. 188, 176 (2007)

[14] G. Pileio and M. H. Levitt, J. Magn. Reson. 191, 148 (2008).

[15] A. K. Grant and E. Vinogradov, J. Magn. Reson. 193, 177 (2008).

[16] W. S. Warren, E. Jenista, R. T. Branca, and X. Chen, Science 323, 1711 (2009).

[17] M. H. Levitt, Spin Dynamics. Basics of Nuclear Magnetic Resonance (Wiley, Chichester, 2001).

[18] R. R. Ernst, G. Bodenhausen, and A. Wokaun, Principles of Nuclear Magnetic Resonance in One and Two Dimensions (Clarendon Press, Oxford, 1987).

[19] D. P. Weitekamp, A. Bielecki, D. Zax, K. Zilm, and A. Pines, Phys. Rev. Letters 50, 1807 (1983).

[20] J. H. Ardenkjaer-Larsen, B. Fridlund, A. Gram, G. Hansson, L. Hansson, M. H. Lerche, R. Servin, M. Thaning, and K. Golman, Prog. Nat. Acad. Sci. 100, 10158 (2003). 